\title{
Investigating ATM System Accessibility for People with Visual Impairments
}

\author{
Okebiro Jared Omari ${ }^{1}$, Omariba Bosire Zachary ${ }^{2}$ \\ ${ }^{1}$ Lecturer University of Kabianga \& PhD. IT student, Masinde Muliro University of Science and Technology, \\ Kenya. \\ ${ }^{2}$ Lecturer Egerton University \& PhD. IT student, Masinde Muliro University of Science and Technology, Kenya.
}

\begin{abstract}
The aim of this paper is to draw attention for the evaluation of the accessibility and usability of Automated Teller Machine (ATM) systems from the point of view of visually impaired persons. It suggests that there is a need for a more precise application of assistive technologies in order to develop user friendly ATMs that will aid effective accessibility without the requirement of assistance by ordinary people who may take advantage of their visual challenges and project them to all odds of insecurity for their cash. The paper suggests that, while ATMs, as opposed to across the counter services, are now a widely accepted means of easing long queues in the banks and as a time saving factor for the banking services to the consumers. An accepted feature of the ATMs is to incorporate assistive technologies which still remain a challenge even with the formative years of ATM applications. It proposes possible contexts that may benefit the visually impaired from far-reaching exploration and incorporation of relevant assistive technologies. The paper should be of interest to financial institutions whose passion is to render better services to their customers in this era of technology considering that there are those customers who wish to transact with them and even need privacy with the ATMs but cannot access such benefits because of their cognitive challenges and specifically the visually impaired. Finally the paper offers a snap-shot of some of the widely-used assistive technologies for the visually impaired when using ATMs and their associated procedures and potential pitfalls.
\end{abstract}

Keywords: accessibility, Automated Teller Machines, Assistive Technology, usability, visually impaired

\section{Introduction}

Since the advent of new Information Communication Technologies, there has been significant surge in ATM assistive technologies. This has made life easier with accessibility of banking services by all but accessibility challenges by the visually impaired have not been fully addressed. An Automated Teller Machine is referred to as a cash machine, a cash dispenser and 'the hole in the wall' among other names. The ATM is an electronic computerized telecommunications device that allows financial institutions (e.g. bank or building society) customers to directly use a secure method of communication to access their bank accounts. Being a self-service banking terminal, ATM's accepts deposits and dispenses cash. Most ATM's also let users carry out other banking transactions such as check balance, pay bill, change PIN, and request a mini-statement. ATM's are activated by inserting a bank debit card into the card reader slot. The card will contain the customers account number and PIN (Personal Identification Number) on the cards magnetic stripe.

When a customer is trying to withdraw cash for example, the ATM calls up the banks computers to verify the balance, dispenses the cash and then transmits a completed transaction notice. This is explored by the developments in information and communications technology (ICT) that support the adoption of software based on the user interface. Generally the paper is coined on the developments in ATMs interfaces and its enhancement on the human machine interaction (usually shortened as HCI) for the blind users. HCI describes the interaction between the user and a computer. But there are also a number of accessibility barriers for those with disabilities and other specific conditions like the blind that are in digital exclusion even with limited available ATM interaction facilities for them. ATMs offer services that are vital in today's life but still the blind, old and people with other cognitive challenges still may have serious accessibility issues which may limit use of such facilities or point of sale systems.

Many different things could be regarded as part of the HCI, like the way the screen looks, or whatever the program makes it clear to the user what they have to do next. This is where the term user friendly originated from. The user interface is becoming a sensitive part of the software system since we have different categories of people using computers. Constant innovation and changing approaches to programming and design have raised issues on user accessibility. Designing interfaces with special users in mind more especially the visually disabled is a challenge. 


\section{Automatic Teller Machines (Atms)}

Cash dispensers and automatic teller machines (ATMs) are a common form of public access terminal, and have been in general use for many years. A number of guidelines, in various countries, have attempted to specify the parameters to alleviate the problems of disabled users. However it is only where there is relevant legislation, that these guidelines have been applied in a consistent manner. Within the European Union there are numerous different specifications required for ATMs, which means that manufacturers have to make slightly different models for different countries which adds to their costs. Although keypads with 1,2,3 in the top row (as on a conventional telephone) and with a raised dot on the 5 key are the norm, the position and marking of the function keys varies from country to country. Many manufacturers offer a range of accessibility options such as:

- Audible prompts or audible output of non-confidential information

- The facility to operate all functions from the keypad so overcoming the need to use a touch screen.

- Providing space for a wheelchair footrest when using a perpendicular approach (rather than a

- parallel approach) to the ATM.

- Illuminated card slots.

- Multi-lingual display screens.

However many purchasers are reluctant to pay extra for such facilities unless they are obliged to do so (e.g. a regulatory or legal requirement). The MeAC study (Assessment of the Status of e-Accessibility in Europehttp://ec.europa.eu/information_society/activities/einclusion/library/studies/meac_study/index_en.htm) found that across the EU countries as a whole on average $8 \%$ of the ATMs installed by the two main national retail banks provided talking capabilities to customers with disabilities. A follow-up study a year later indicated that there had been no significant change over the intervening period. The guidelines issued by banking organizations are frequently ignored by the ATM suppliers since they feel them to be un-implementable or inappropriate. For instance the force to operate a key is quoted in a number of guidelines as not to exceed 22.2 Newton; many experts feel this figure ought to be an order of magnitude lower.

The visually impaired person may encounter a lot of difficulties when using ATMs such as, learning where the objects are located on the screen, accidentally deleting a file or withdrawing money from the ATM. Improving interface navigability for visually impaired users has been dealt with in S. Kane, et al. (2009) through a set of gestures which allow the user to move around the UI controls and listen to their contents. Commercial implementations of this interface now exist as reported by Apple Inc. However, concerning text entry, blind users are still forced to use the built-in on-screen keyboard. Even though this keyboard may be able to speak the character above which the user's finger is located, typing using this method is time consuming and error-prone. Letters on such keyboards are tiny and are placed too close to one another due to the constrained screen size and so the plethora of touch targets makes typing slow to the point of frustration.

\section{Visual Impairment}

Many things can cause visual impairment. Sometimes, it can be treated, and sometimes it cannot. Impairment is a functional deficit compared to what is commonly viewed as "the norm". Impairments are usually decomposed into one of the classifications motor, sensor, and cognition. To better understand visually impaired persons, knowledge of the cause of the impairment should be known. Some of the major reasons are cataracts, macular degeneration, glaucoma, trachoma, diabetic retinopathy, and other retinal disorders Microsoft Encarta (2000). Users have struggled over years to interact with machines or systems in terms of accessing information, processing information or getting a task done. Initial user interfaces have not made all this easy. The cognitive challenges on the human beings have also contributed. Like one being visually impaired, the terms 'visually impaired person' and 'blind' are the subjects of great confusion, among sighted and visually impaired people alike Martin, (1997).

In Kenya, the constitution has catered for equality on all people and The Commonwealth Disability Discrimination Act 1992 (DDA) makes it unlawful to discriminate against a person on the grounds of a disability. We are experiencing instances in which the Visually Impaired have been discriminated technologically in that most of them have not been in a position to access banking services on their own other than being assisted which is very risk as they can be prone being coned and all. They have been digitally excluded from some essential services like locating ATM locations on their own, checking their balances, withdrawals, deposits printing statements, card insertion, PIN entry among other services.

According to the definition and classification of visual impairments as stated by Silvia D. et al (2006), the visual function consists of different specific perceptual abilities, among which are:

- Visual acuity (or visus), the capacity of focusing on details, both from far way and nearby;

- Visual field dimension, the amplitude of the space one can embrace at a glance;

- Chromatic sense, the perceptual capacity allowing color distinction; 
- Contrast sensitivity, the capacity to perceive the difference between two elements located side by side;

- Light sensitivity, the eye reaction to light and light variations;

- Stereopsis and binocularity, the possibility or capacity to perceive depth on the basis of the difference in points of view of the two eyes.

Each of these abilities concurs, to some extent, to make a person achieve the best possible visual perception, but visual acuity and visual field dimension are generally recognized as the two fundamental parameters. Silvia D. et al (2006), states that there are many other problems affecting the visual function, such as difficulty in perceiving reliefs, lack of resistance to dazzle, reduced ability to follow movements ("nystagmus") which can produce involuntary, rhythmical, repeated oscillations of one or both eyes.

\section{Digital Exclusion Of The Visually Impaired}

Information and communication technology (ICT) is at its cutting edge and key for everyday life, facilitating access to essential services such as those of the banking sector. ATMs have made it possible for bank transactions but the blind have not fully embraced their utilization compared to the normal users. Assistive technology is anticipated to empower and improve independence for such a category of people so that they are not prone to double-cross at any time they are interacting with such point of sale systems.

ATMs have are such useful and they came in to rescue bank customers from the long queues and all. But amid all the benefits they come with, they too can be frustrating depending on their design and structure during usage. Standard ATMs for any other person have their challenges but for the blind too, they experience the following challenges and inconveniences:-

- Inability to locate the ATM. This is due to the fact that they cant see or even tell the corners where the systems are installed.

- Inability to see the ATM screens clearly. This also can be due to the location of the ATM .It is even difficult for them view the contents of the ATM menu on the screen.

- Waiting in the queue for long hours due to breakdowns or network problems from the central bank server. If the other users in the queue experience difficulties in using the machine because of their inability, this will mean you also waiting a little bit longer in the queue.

- With their inability to see, the visually impaired users will now and gain use trial methods inserting the ATM card which they can repeat doing wrongly. This problem remain more common with blind ATM users since they are not familiar with their ATM cards card and cant even see how they look other than touch and feel.

- Getting money from the dispenser. Because of the same inability that they don't see, the blind users will struggle to collect their money after dispensation.

- Because most ATM screens have been designed to be used by the normal user with no cognitive challenges, the blind may not perform some operations. Generally the blind users will find the instructions on how to perform operations quite difficult to understand. Even on mastering the interactive screen, the blind users may find different ATMs with different menu options whose arrangement is opposite to the corresponding menu keys.

- In an event that the visually impaired requires further transactions, such as check balance or print statement or even top-up a mobile phone it will even be more difficult for such a person given that there are no such straight provisions.

- The visually impaired may have to wait stranded in an event that the ATM swallows the card.

- The visually impaired will always find it difficult entering of keying in PIN numbers, since some ATMs may lack Braille systems as well as choosing the transaction option may also remain a challenge.

\section{Assistive Technologies}

According to Kristin S., Till H. (2010) Assistive Technology, is a technology both hardware and software, with the meaning to assist the user in order to reach a particular goal, typically tailored to her/his special needs, such as blindness. Assistive Technology consists of devices or services that help people to achieve greater independence. The England Assistance Technology Act of 1998 (defines an assistive technology device as "...any item, piece of equipment or product system, whether acquired commercially off the shelf, modified or customized, that is used to increase, maintain, or improve functional capabilities of individuals with disabilities." With the advent of new assistive technologies, the potential to access information by the blind has been critical and vital. Disappointingly, challenges to accessing these technologies remain. Blind and partially sighted people face unpleasant impediments, impacting on their use and enjoyment of ATM usage. Many a time they require to be assisted which cancels the whole logic of advent of such technologies. 
Inspired by the demands and challenges experienced by the visually impaired customers, assistive technology has been instrumental in improving and simplifying how such challenged people access banking services. Changes in access to withdrawal, balance checking, depositing, transfer of money, entering PIN, choosing transaction option among others have been greatly improved by incorporating assistive technology into the ATMs. Some assistive technologies have many settings; for example, screen magnification software provides multiple options for zoom, color, contrast, cursor, and more.

\section{Assistive Technology Devices}

Assistive technology devices can be simple or complex, depending upon the needs of the individual. Examples include:

- Aids for Daily Living - Self help aids for use in activities such as bathing, cooking, dressing, eating, and home maintenance

- Communication Aids - Devices for people with limited or no speech including augmentative communication devices and electronic and manual communication boards.

- Educational Aids - Devices that allow people with disabilities to perform work or school-related activities. Examples are input and output devices, modified or alternate keyboards, and special software (including speech recognition software) that allow people with disabilities to use a computer.

- Mobility Aids - Devices to allow freer movement which include patient lifts, transfer aids, and all types of wheelchairs and three-wheel vehicles.

- Prosthetics and Orthotics - Artificial limbs, braces or other devices to augment, replace, or substitute for missing or non-functioning body parts.

- Sensory Aids - Devices to help people with visual or hearing disabilities such as hearing aids, lowvision aids, and telecommunication devices for the deaf.

The blind people cannot exhaustively use computers and thereby automated machines because of two basic problems and for those reasons they need the aid of assistive technologies.

i. Inability to use normal data input devices like keyboards \& mice as effectively.

ii. Inability to view the output on computer screen.

\section{Usability}

Usability denotes the usefulness of a product or service and the ease with which people can use it; but it remains a secondary concern in software development Singh and Kotze (2003). Highman (2001) thought there was a need to persuade designers to think harder about usability i.e. to understand and identify user expectations, needs, characteristics, limitations as well as the task functionality and environment. Usability is also important because the public will not invest time and effort to learn the system, they do not want training, and rarely read manuals. The traditional models for software development including object-oriented approaches and agile methods do not in general clearly identify a role for interaction design in systems development. Interaction design is the process of designing interactive products to support people in their everyday social and work lives. In particular, it is about creating user experiences that enhance and extend the way people work, communicate and interact Preece, Rogers et al. (2002). Counterpoint (2001) found that subjects had different levels of training on delivery e.g. some people were just shown the electronic program guide (EPG) whereas others were introduced to all the relevant services. Some people only sought the range of channels available but did not investigate other services. Sometimes new services were introduced by another person. When functions were found by accident, the mistake was learnt so it could be redone when needed. Many people learnt to use the service by trial and error as Counterpoint (2001) note, finding services by accident or remaining unaware that they had interactive options available. These reactions illustrate unintuitive design.

Several researchers have produced sets of generic usability principles which can be used in improving software for example, Dix et al. (1998); Nielsen (1993); Nielsen (2000); Cato (2001); Mayhew (1999); Preece et al. (1994); Shneiderman (1998); Shneiderman (2000); Thimbley (1990)). Some of these principles relate to learnability, visibility, consistency and standards, flexibility, robustness, responsiveness, feedback, constraints, mappings, affordances, stability, simplicity, help and documentation. Unfortunately the definitions of the design and usability principles are mostly too broad or general, and in some cases very vague, to be directly and seamlessly applied in a particular context Universal design can increase the user experience concerning the use of a particular product or service considerably, meaning that a solution becomes more accessible to ideally all potential users with a different background, such as skills, knowledge, age, gender, disabilities, literacy, etc. gender, disabilities, literacy, etc. A central issue in universal design of ICT solutions is the development of flexible multimodal user interfaces that can meet a user's different needs, abilities, situations, preferences, and devices Fuglerud (2009). 


\section{Accessibility}

In the context of ICT, accessibility describes the degree to which a solution is accessible by as many people as possible, in particular those with impairments. It is always a challenge to design a product or system so that all potential users are able to access it. In other words, it is of great concern not to exclude large user groups such as people with disabilities. However, it is our impression that research about how to design accessible ID-technology seems to be very limited. This is also the conclusion of Sauer et al. (2010) who reports that the number of studies regarding accessibility and security is extremely limited. According to Hitchcock, C., Stahl, S.(2003), the computer can be considered, with good reason, an effective tool for visually impaired persons, as it contributes to counterbalance their disability and allows them to perform a host of activities which otherwise might be very difficult.

Computers open interesting perspectives for all users with disability, in that they provide access to reading and writing of all kind of and several alternative output-tools are often tried out in order to choose the one that best fits the user's individual requirements (e.g., a user may prefer an enlarging program while another may opt for a screen reader). Sometimes, in order to meet the specific needs of people with visual impairments, it is important to customize some computer/software features. For instance, the user may need to set the size of the mouse pointer, icons, menus and texts. If this is not sufficient, special software can be added, such as a screen reader with text-to-speech capabilities, or magnifying programs. While in most cases these programs allow users to use the computer autonomously, they are often incompatible with online applications. Many of the latest ATM software products are mainly based on text communication and may therefore pose genuine difficulties for low vision users. Even texts can be difficult to read for such users if special fonts are used, and enlarging software doesn't automatically solve all problems.

Summarizing, in the light of the above considerations, the computer can be considered as an effective tool for visually impaired users, but there is still a need to focus on the real usability of ATM systems. "Is computer based ATM system accessible to visually impaired users? Can visually impaired users use the same software as their colleagues"? Answering these questions entails an evaluation of the software tools commonly used in banking, including ATM software, from the point of view of their usability and accessibility. The visually impaired for the ATM system should be trained to use the system to fully benefit from the ATM potential.

However, looking carefully at some characteristic features of ATM software, it becomes immediately evident that products' interfaces often poses problems for low vision users, due to small characters, and confusing backgrounds. As a result, the banking effectiveness of these products appears to be limited, and unfortunately assistive technologies are not always sufficient to increase software usability. As a consequence, this ATM software proves to be a product of low "accessibility" for most users, particularly for those who have problems of contrast sensitivity and visual acuity. Even with the assistive technologies incorporated, when considering other interfaces of the interface, it cannot be concluded that the product is absolutely fine for visually impaired users. But if the background should offer a higher degree of contrast to narrow the space to be eye-controlled, then the system can be fruitful by the visually impaired uses especially those with a narrow visual field as an exercise for visual stimulation. Screen magnification software in this specific case would diminish its accessibility, since it decreases the amount of the screen one can see at a glance

\section{Security Gaps}

According to the European Blind Union (EBU), in their paper "Response to European Commission Green Paper 'Towards an Integrated European market for card, internet and mobile payments' “ (2012), From a blind or partially sighted person's perspective there are significant security gaps in physical transactions because they have to rely on staff to enter the correct amount on the PIN Entry Device. Most people with sight loss cannot see well enough to read what is on the screen and therefore verify the amount being requested. There is also an issue with the option to add a tip sometimes encountered in restaurants. As a result, physical transactions are not secure and people with sight loss are put in a vulnerable position.

In addition, people with sight loss have no way of knowing whether or not the PIN Entry Device is a genuine payment terminal or a fraudulent one which will steal their card details. As such visually impaired people have to place trust in the person taking the money; they have to trust that others have entered the amount correctly and have not taken additional money, either maliciously or by accident.

In this paper the use of biometric features will be a solution to eliminate some of the security flaws that can emerge whenever the visually impaired persons are using the ATM systems. John G. (2009), states that a biometric is a physical or behavioral feature or attribute that can be measured. It can be used as a means of proving that you are who you claim to be, or as a means of proving without revealing your identity that you have a certain right. 
Biometrics which is commonly used to confirm identity include: Fingerprint recognition, Iris recognition, Face recognition, Hand geometry recognition, and Vein recognition. Other biometric modalities which have been developed but are not currently used in self-service terminals in Kenya include: Voice recognition, Gait, Ear shape, Retina patterns, and DNA

A biometric system is essentially a pattern recognition system that operates by acquiring biometric data from an individual, extracting a feature set from the acquired data, and comparing this feature set against the template set in the database. Some physiological and medical factors can affect the usability and efficiency of biometrics. The obvious advantage of biometric systems is that the user no longer has to remember PINs (personal identification numbers) and keep this number secret. People with a cognitive impairment will find most biometric systems easier to use and provide a greater level of security. People who have limited or no use at all of arms or hands will find using face and iris recognition systems an advantage as they will not have to swipe a card or type in a name or PIN number.

\section{Conclusion}

People with disabilities encounter daily many barriers that prevent them to interact with electronic devices in their surroundings. When the government agencies or commercial sector does not provide the necessary services or facilities, it may be necessary to consider introducing mandatory regulation or legislation. In the area of accessibility of self-service terminals, the market is not providing terminals which the user organizations deem to be acceptable in terms of accessibility. However the introduction of mandatory requirements necessitates that there are a clear set of guidelines which cover all relevant types of terminal and do not limit manufacturers from introducing new ways of improving accessibility. A priority is to develop a single common standard for accessibility across the different application sectors so that manufacturers and service providers are clear as to what they are expected to provide. The next stage would be to legislate that this standard is mandatory. Therefore the financial institutions will not be left with an option to procure ATM systems which are inaccessible to the visually impaired. Finally it is seen through this paper that the incorporation of biometric features will be essential to ensure that these systems are secure enough.

\section{References}

[1]. Adams, A. S. and Thieben, K. A. (1991), Automatic teller machines and the older population. Applied Ergonomics, 22,85 -90.

[2]. Miller, A.W, 2006 [Online], Who invented the ATM machine? Automated Teller

[3]. Most, .M.M., Craddick, S., Crawford, S., Redican, S., Rhodes, D., Rukenbrod, F., Laws, R. (2003). Dietary quality assurance processes of the DASH-Sodium controlled diet study. Journal of the American Dietetic Association, 103(10): 1339-1346.

[4]. Preece, J., Y. Rogers, et al. (2002). Interaction Design: Beyond human-computer interaction. New York, USA, John Wiley \& Sons.

[5]. Singh, S. and P. Kotze (2003). An Overview of Systems Design and Development Methodology with Regard to the Involvement of Users and Other Stakeholders. SAICSIT 2003: IT Research in Developing Countries, Fourways, South Africa, SAICSIT.

[6]. Whitney, C.W., Lind, B.K., Wahl, P.W. (1998). Quality assurance and quality control in longitudinal studies. Epidemiologic Reviews, 20(1): 71-80.

[7]. EBU ( April 2012), Response to European Commission Green Paper 'Towards an Integrated European market for card, internet and mobile payments'

[8]. Higham, 2001. N. Designing TV for Granny. http://news.bbc.co.uk/hi/english/entertainment/tv_and_radio/newsid_1689000/1689552.stm

[9]. Counterpoint. 2001. Digital Television - Consumers' Use and Perceptions: A Report on a Research Study See at:. www.oftel.gov.uk/publicaitons/research/2001/digtv0901.pdf

[10]. Silvia Dini, Lucia Ferlino, Anna Gettan, Cristina Martinoli, Michela Ott (2006) Educational software and low vision users: evaluating accessibility factors

[11]. Riel, M. (1994), Educational change in a technology-rich environment. Res. Comput. Edu. J. 26(4), 452-474

[12]. Hitchcock, C., Stahl, S. (2003), Assistive technology, universal design, universal design for learning: improved learning opportunities. Spec. Edu. Technol. J. 18 (4).

[13]. Kristin S.F, and Till H.R (2010), Review of Literature on Usability and Accessibility Issues of Personal Identification Management Systems.

[14]. G. Sauer, J.Holman, J. Lazar , H. Hochheiser, J. Feng (2010), “Accessible privacy and security: a universally usable humaninteraction proof tool ", Univ Access Inf Soc 9:239-248, Springer

[15]. K. S. Fuglerud, A. Reinertsen, L. Fritsch et al. (2009), "Universal design of IT-based solutions for registration and authentication", Norwegian Computing Center, Oslo,

[16]. J. Nielsen (2000), "Security \& Human Factors,", http://www.useit.com/alertbox/20001126.html. 27.11.2012

[17]. S. Kane, C. Jayant, J. Wobbrock, and R. Ladner. (2009) Freedom to roam: a study of mobile device adoption and accessibility for people with visual and motor disabilities. In Proceedings of the 11th international ACM SIGACCESS conference on Computers and accessibility, pages 115-122. ACM,

[18]. Inc. Apple - Accessibility - Voiceover - In Depth. http://www.apple.com/accessibility/ voiceover/, September 2013.

[19]. Europehttp://ec.europa.eu/information_society/activities/einclusion/library/studies/meac_study/index_en.htm) July 2013.

[20]. John Gill, (2009) The Markets for the Adaptation of Self-Service Terminals to be Accessible by People with Disabilities. 